\title{
Pyrogenic vorticity from windward and lee slope fires
}

\author{
J.J. Sharples ${ }^{\text {a }}$, A.E. Kiss ${ }^{\text {ab }}$, J. Raposo ${ }^{\mathrm{c}}$, D.X. Viegas ${ }^{\mathrm{c}}$ and C.C. Simpson ${ }^{\mathrm{a}}$

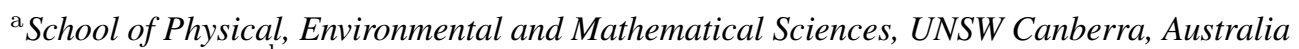 \\ ${ }^{\mathrm{b}}$ ARC Centre of Excellence for Climate System Science \\ ${ }^{\mathrm{c}}$ ADAI, University of Coimbra, Portugal
}

Email: j.sharples@adfa.edu.au

\begin{abstract}
Research into dynamic bushfire behaviour conducted over the last few years has shown that bushfires burning on lee-facing slopes can exhibit atypical forms of propagation under extreme weather conditions. For instance, recent numerical simulations have indicated that pyrogenic vorticity (the curl of the velocity field) is a key driver of rapid lateral fire spread - as a consequence, this type of fire propagation is being referred to as Vorticity-driven Lateral Spread (VLS). The VLS phenomenon has also been reproduced in laboratory-scale experiments, some of which show clear vortex structures. The presence of vorticity in the experiments and simulations suggests that additional insights into the physical processes may be drawn from direct examination of the fluid dynamical equation governing vorticity. We neglect Coriolis effects and divergence but do not make the Boussinesq approximation, giving the governing vorticity equation:
\end{abstract}

$$
\frac{D \boldsymbol{\omega}}{D t}=(\boldsymbol{\omega} \cdot \nabla) \mathbf{u}+\frac{1}{\rho^{2}} \nabla \rho \times \nabla p+\nabla \times \mathbf{F}
$$

where $\mathbf{u}$ is the velocity field, $\boldsymbol{\omega}=\nabla \times \mathbf{u}$ is the vorticity, $\rho$ is the density, $p$ is the pressure and $\nabla \times \mathbf{F}$ represents eddy viscosity and body forces.

In this paper we provide a rudimentary theoretical analysis of the vorticity equation for the separate situations of fires burning on leeward and windward sides of a triangular ridge under the influence of a strong cross-wind and assumed separation of the flow in the lee of the ridge. We consider the behaviour of the right and left flanks of the fire with some simplifying assumptions about the effects of the fire on the local wind flows.

The analyses indicate that on the leeward slope the fire should produce pyrogenic vorticity in the vertical direction due to tilting of ambient lateral vorticity (due to the separated boundary layer) by the plume of the fire. As vertical vorticity is produced it is enhanced through stretching by the accelerating buoyant updraft. Longitudinal vorticity is generated in the downwind direction by tilting of the vertical vorticity in addition to the presence of baroclinic torques caused by pyrogenic differences in density of the flow.

The generation of pyrogenic vorticity over a fire on a lee slope arises due to the fact that separation of the flow in the lee of the ridge creates a sheet of vorticity at some height above the lee slope. This region of ambient vorticity occurs at a height where the pyrogenic velocity gradients are large enough to drive interactions that result in tilting of the ambient vorticity. For the windward fire case, the ambient vorticity only occurs close to the surface where the pyrogenic velocity gradients required to tilt the ambient vorticity are negligible. So while some longitudinal vorticity can arise through baroclinic forcing, the case of a windward fire is far less likely to produce the strong vertical vorticity required to drive the VLS phenomena. The same conclusions will also hold for fires burning on flat ground.

While these analyses should be considered as preliminary and approximate, they do provide some useful insights into the dynamics of the VLS phenomenon. For instance they provide a physical explanation of why the VLS phenomenon occurs exclusively in connection with steep, lee-facing slopes, or with lee slopes that possess features such as sharp bluffs, which act to promote flow separation. Moreover they demonstrate the importance of the various driving factors, namely tilting of ambient lateral vorticity, stretching of vertical vorticity by Lagrangian acceleration of the buoyant plume and baroclinic forcing arising due to the effect of the heat of the fire on the density of the flow.

Keywords: Pyrogenic vorticity, extreme fire behaviour, VLS, atypical lateral spread, fire-environment interactions 


\section{INTRODUCTION}

Southeastern Australia has experienced a number of serious bushfire incidents over the last decade or so, with many of these incidents occurring in rugged terrain (Sharples et al., 2012; Cruz et al., 2012). This pattern has been echoed in other regions around the globe (Peterson et al., 2015). As such, there has been considerable interest in how these fires interact with the airflow over hilly terrain. One particular area of interest concerns the prevalence of terrain-induced turbulence, such as occurs when air flows over steep terrain and separates from the surface to form eddies or rotors over leeward aspects (Byron-Scott, 1990; Wood, 1995). Wood (1995) argued that if winds were sufficiently strong, then eddies could be expected to form over leeward slopes inclined at about $20^{\circ}$ or more regardless of atmospheric stability conditions. Sharples et al. (2010) also demonstrated the prevalence of lee rotors over slopes exceeding $20-25^{\circ}$ when wind speeds were above about $20 \mathrm{~km} \mathrm{~h}^{-1}$.

Byron-Scott (1990) presented a two-dimensional analysis of the interaction between a lee rotor and pyrogenic buoyancy, employing the first circulation theorem of physical hydrodymanics (Holton and Hakim, 2012) as the primary theoretical tool to derive an equation for the rotor motion of an air parcel around a closed triangular curve over a lee slope. Through consideration of analytical solutions of his rotor equation Byron-Scott (1990) concluded that the presence of a lee slope fire could enhance lee rotor motions, and that vigorous lee rotors could contribute momentum to the main airflow above them, with consequences for subsequent fire development.

However, the analysis of Byron-Scott (1990) should only be considered as approximate due to its twodimensional nature. As discussed by Simpson et al. (2013), rotors that form in the lee of three-dimensional terrain are more transient in nature: they have a cellular structure and are intermittently shed downwind. So while the permanent two-dimensional rotor assumed by Byron-Scott (1990) does provide important insights into the physical interactions between terrain-modified winds and an active fire, it should not be considered as totally realistic.

Simpson et al. (2013) used a numerical model to show that in the full three-dimensional setting, a fire burning on a leeward slope actually produces vertical vorticity, and that this vertical vorticity promotes lateral fire propagation across the slope in a process that has been termed vorticity-driven lateral spread (VLS). Vertical vorticity is also evident in vortex structures observed in laboratory experiments involving fires on lee-facing slopes. Figure 1 shows an example of a fire whirl produced by an experimental lee slope fire: both vertical and longitudinal vorticity are evident in the photograph. The enhanced likelihood of fire whirls forming on leeward slopes was first noted by Countryman (1971).

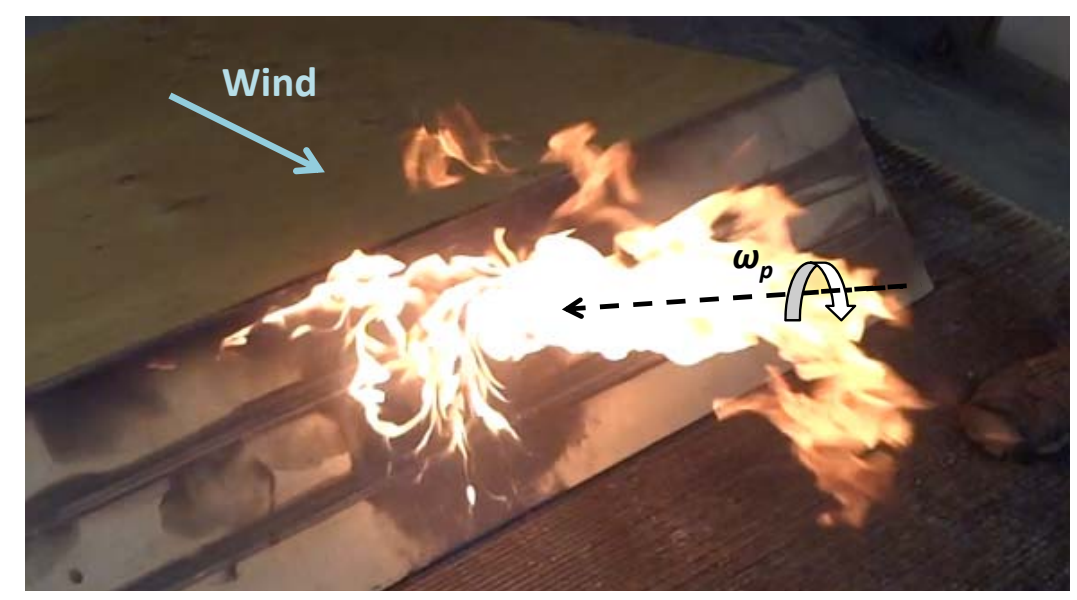

Figure 1. Experimental fire in a wind tunnel showing a fire whirl (vortex) on the leeward slope of an idealised ridge. Note that the vortex is on the left flank of the fire and has components $\omega_{x}, \omega_{z}<0$. The pyrogenic vorticity $\boldsymbol{\omega}_{p}$ and its orientation are indicated in the figure.

The analysis presented by Simpson et al. (2013) provides a physical explanation for the atypical lateral spread observed in a number of fires (Sharples et al., 2012; Raposo et al., 2015), but doesn't allow any direct insight into the origin of the vertical vorticity that apparently drives the lateral spread. Therefore the question naturally arises as to whether it is possible to conduct a theoretical analysis similar to that of Byron-Scott (1990), but 
which acknowledges the full three-dimensional nature of the problem. Moreover, Simpson et al. (2015) used numerical modelling to demonstrate that VLS also occurs when a windward fire moves across a ridge onto the lee slope.

This paper presents a rudimentary analysis based on the vorticity equation, which follows along the lines of analyses presented by Jenkins et al. (2001) in the context of fire plume rotation, and by Klemp (1987) in the context of tornadic thunderstorms. We begin by considering fires burning on leeward slopes and examining the drivers of vorticity under a number of simplifying assumptions about the effects of the fire on the local wind flows. We also consider fires burning on windward slopes under similar assumptions to test the hypothesis that the mechanism underlying the generation of vertical (and longitudinal) vorticity is the same for windward and lee slope fires.

\section{VORTICITY EQUATION AND ANALYTICAL SET-UP}

For a fluid with velocity vector field given by $\mathbf{u}$, the vorticity is defined as its curl: $\boldsymbol{\omega}=\nabla \times \mathbf{u}$. Taking the curl of the momentum equation of $\mathbf{u}$, and neglecting divergence and Coriolois effects, yields the vorticity equation (Vallis, 2006):

$$
\frac{D \boldsymbol{\omega}}{D t}=(\boldsymbol{\omega} \cdot \nabla) \mathbf{u}+\frac{1}{\rho^{2}} \nabla \rho \times \nabla p+\nabla \times \mathbf{F},
$$

where $\rho$ is the local fluid denisty, $p$ is the local pressure, $\nabla \times \mathbf{F}$ represents eddy viscosity and body forces, and

$$
\frac{D \boldsymbol{\omega}}{D t}=\frac{\partial \boldsymbol{\omega}}{\partial t}+(\mathbf{u} \cdot \nabla) \boldsymbol{\omega}
$$

is the material derivative of $\boldsymbol{\omega}$.

In this preliminary treatment, we assume the presence of ambient horizontal vorticity generated by $\nabla \times \mathbf{F}$ within the viscous boundary layer, but we focus on the subsequent evolutions of this vorticity away from the boundary, where we assume viscosity is negligible. In this region gravity is the only body force acting on fluid parcels, so that $\nabla \times \mathbf{F}=\mathbf{0}$. Inclusion of viscous forces would be an interesting extension of the present work, but for now we consider the vorticity equation as:

$$
\frac{D \boldsymbol{\omega}}{D t}=(\boldsymbol{\omega} \cdot \nabla) \mathbf{u}+\frac{1}{\rho^{2}} \nabla \rho \times \nabla p .
$$

In this paper we consider the vorticity generated by the fluid flow over an isolated ridge in the presence of a cross-wind and a heat source (e.g. a fire) on either the windward or leeward slope. We consider equation (3) in terms of the right-handed coordinate system $(x, y, z)$, with the $x$-axis pointing in the downwind direction and the $z$-axis pointing vertically upwards. We express the velocity field in terms of the components $\mathbf{u}=$ $\left(u_{x}, u_{y}, u_{z}\right)$ and the vorticity field as $\boldsymbol{\omega}=\left(\omega_{x}, \omega_{y}, \omega_{z}\right)$. The $x, y$ and $z$ components of equation (3) are as follows:

$$
\begin{aligned}
\frac{D \omega_{x}}{D t} & =\omega_{x} \frac{\partial u_{x}}{\partial x}+\omega_{y} \frac{\partial u_{x}}{\partial y}+\omega_{z} \frac{\partial u_{x}}{\partial z}+\frac{1}{\rho^{2}}\left(\frac{\partial \rho}{\partial y} \frac{\partial p}{\partial z}-\frac{\partial \rho}{\partial z} \frac{\partial p}{\partial y}\right) \\
\frac{D \omega_{y}}{D t} & =\omega_{x} \frac{\partial u_{y}}{\partial x}+\omega_{y} \frac{\partial u_{y}}{\partial y}+\omega_{z} \frac{\partial u_{y}}{\partial z}+\frac{1}{\rho^{2}}\left(\frac{\partial \rho}{\partial z} \frac{\partial p}{\partial x}-\frac{\partial \rho}{\partial x} \frac{\partial p}{\partial z}\right) \\
\frac{D \omega_{z}}{D t} & =\omega_{x} \frac{\partial u_{z}}{\partial x}+\omega_{y} \frac{\partial u_{z}}{\partial y}+\omega_{z} \frac{\partial u_{z}}{\partial z}+\frac{1}{\rho^{2}}\left(\frac{\partial \rho}{\partial x} \frac{\partial p}{\partial y}-\frac{\partial \rho}{\partial y} \frac{\partial p}{\partial x}\right) .
\end{aligned}
$$

\subsection{Flow over an isolated ridge}

We consider an isolated ridge such as the one shown in Figure 2, where we assume that the ridge extends a considerable distance into and out of the page (i.e. along the $y$-axis). The figure depicts a wind blowing from right to left and a fire burning on the leeward slope of the ridge. The flow has separated from the surface in the lee of the ridge, resulting in a wind reversal on the lee slope. Representative vertical wind profiles, assuming no-slip boundary conditions, are shown on the windward slope, at the separation point at the top of the lee slope and further down the lee slope. The light blue shading indicates the region of positive vorticity generated on the windward side by boundary layer shear in $u_{x}$, and extending at some height above the ground on the leeward side as a positive vorticity sheet due to the separated flow. The red arrows indicate the orientation of this lateral vorticity $\omega_{y}$. 


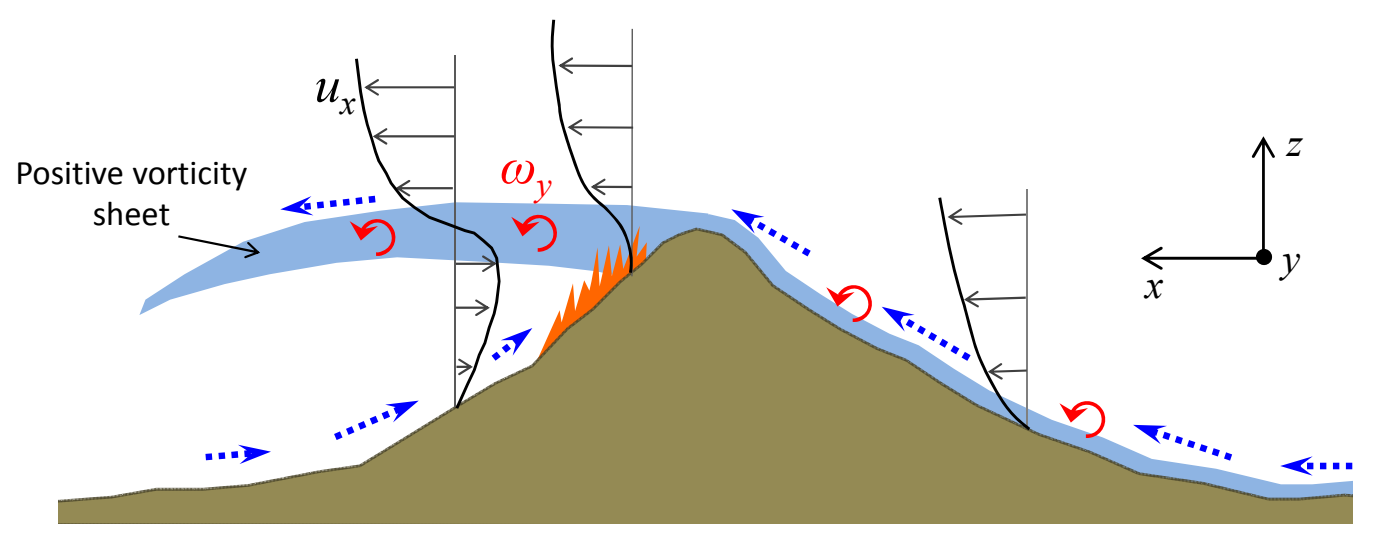

Figure 2. Flow over an isolated ridge with the flow moving from right to left. Representative vertical profiles of $u_{x}$ are shown along with the region of positive lateral vorticity $\omega_{y}$ (light blue shading).

\subsection{Fire on a leeward slope}

With reference to Figure 2, we consider a fire burning on the leeward slope near the top of the ridge. Lateral vorticity generated near the surface on the windward slope through turbulent flux is transported up and over the ridge and persists at some height above the lee slope in the separated flow as the sheet of positive vorticity shown in the figure. Numerical simulations of the flow over a sharp ridge, conducted by Simpson et al. (2013), indicated that this sheet of positive vorticity was present regardless of whether a fire was burning on the leeward slope. Moreover, their simulations indicated that the top hundred metres or so of the lee slope also experienced distinct positive vorticity; this effect is illustrated schematically in Figure 2.

In what follows we consider the vorticity dynamics arising in the region near the top of the leeward slope, and on the flanks of the fire as it burns up the lee slope. The fire creates a buoyant updraft of finite width over the fire area. Figure 3 shows the lateral profile of $u_{z}$ produced by the fire. The buoyant updraft accelerates from zero at the surface to a significant speed at height that depends on the intensity of the fire. Considering the values of the lateral derivative of $u_{z}$ at a height that coincides with the region of positive lateral vorticity $\omega_{y}$, the figure indicates the following conditions:

$$
\frac{\partial u_{z}}{\partial y}<0 \text { (left flank), and } \frac{\partial u_{z}}{\partial y}>0 \text { (right flank). }
$$

The heat of the fire results in a reduction in the density of the air above it. This produces a similar picture to that shown in Figure 3, but with the density profile curves inverted compared to $u_{z}$; that is, density is larger away from the fire and smaller above the fire. The lateral derivatives of the density on the flanks of the fire, in the region coinciding with $\omega_{y}>0$, therefore satisfy:

$$
\frac{\partial \rho}{\partial y}>0 \text { (left flank), and } \frac{\partial \rho}{\partial y}<0 \text { (right flank). }
$$

With reference to Figure 2, the vertical profile of $u_{x}$ on the leeward side indicates that $u_{x}$ is increasing with height throughout the region characterised by positive lateral vorticity. Moreover, with reference to Figure 3, the vertical velocity will be non-decreasing with $z$ throughout the vertical profile due to Lagrangian acceleration of the plume. Although the density will be increasing slightly with height as the buoyant plume entrains cooler air, we regard this change as negligible in the current analysis. Similarly we assume that any changes in density due to downwind entrainment of cooler air are negligible. We therefore assume the following conditions hold on both flanks of the fire:

$$
\frac{\partial u_{x}}{\partial z}>0, \quad \frac{\partial u_{z}}{\partial z}>0, \quad \frac{\partial \rho}{\partial z} \approx 0, \text { and } \frac{\partial \rho}{\partial x} \approx 0 .
$$

We assume the usual decrease in pressure with altitude, but will assume that changes in pressure in the downwind and lateral directions are negligible, so that on both flanks of the fire we have:

$$
\frac{\partial p}{\partial x} \approx 0, \quad \frac{\partial p}{\partial y} \approx 0 \text { and } \frac{\partial p}{\partial z}<0 .
$$




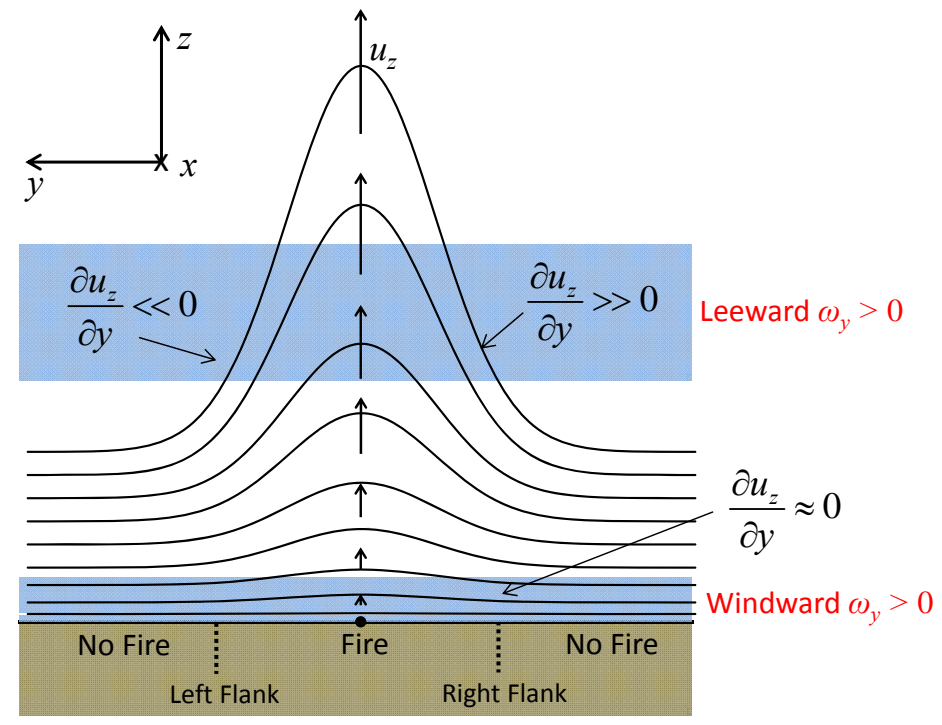

Figure 3. Schematic illustration of the lateral variation in the vertical velocity $u_{z}$ at different heights, caused by the pyrogenic updraft over the fire. The schematic shows the downwind-looking view (positive $x$-axis points into the page), with a fire assumed to be burning in the centre of the lateral profile. The contours represent the variation of $u_{z}$ with $y$ at various heights $z$; these are offset vertically for clarity. The blue shading indicates the regions of strong positive lateral vorticity for the windward and leeward cases.

Byron-Scott (1990) notes the possibility of an intense lee slope fire enhancing the speed of the separated flow through entrained momentum. If such a possibility was realised this would also imply the following conditions:

$$
\frac{\partial u_{x}}{\partial y}<0 \quad \text { (left flank), and } \frac{\partial u_{x}}{\partial y}>0 \quad \text { (right flank) }
$$

otherwise this term could be negligible on both flanks.

Finally, we assume that on both flanks

$$
\frac{\partial u_{z}}{\partial x} \approx 0 \text { and } u_{y} \approx 0, \text { which implies } \frac{\partial u_{x}}{\partial x} \approx-\frac{\partial u_{z}}{\partial z}<0, \text { assuming quasi-continuity. }
$$

Incorporating conditions (7-12) into equations $(4,5,6)$ we obtain the system of equations on both flanks:

$$
\begin{aligned}
\frac{D \omega_{x}}{D t} & =\omega_{x} \frac{\partial u_{x}}{\partial x}+\omega_{y} \frac{\partial u_{x}}{\partial y}+\omega_{z} \frac{\partial u_{x}}{\partial z}+\frac{1}{\rho^{2}} \frac{\partial \rho}{\partial y} \frac{\partial p}{\partial z} \\
\frac{D \omega_{y}}{D t} & =0 \\
\frac{D \omega_{z}}{D t} & =\omega_{y} \frac{\partial u_{z}}{\partial y}+\omega_{z} \frac{\partial u_{z}}{\partial z}
\end{aligned}
$$

We note that the magnitudes of $\partial_{y} u_{z}$ and $\partial_{y} \rho$ will depend on the intensity of the fire: more intense fires will produce larger magnitudes on both flanks. We also note that $\left|\partial_{x} u_{x}\right|<\left|\partial_{z} u_{z}\right|$ due to gas generation and density reduction in the plume, hence the assumption of quasi-continuity in (12). However, we have assumed such non-conservation of volume is negligible in the vorticity equation (3).

\subsection{Fire on a windward slope}

The key difference between the case of a fire on the windward slope and on the leeward slope is that in the immediate lee of the ridge the separation of the flow places the region of positive lateral vorticity at some height above the surface. In contrast, on the windward slope positive vorticity is constrained to a very thin layer close to the surface. This effect can be seen in the numerical simulations of Simpson et al. (2013), for example. 
Figure 3 indicates that in the leeward fire case the region of positive lateral vorticity coincides with large values of $\left|\partial_{y} u_{z}\right|$. In contrast, near the surface on the windward side where $\omega_{y}>0$, the lateral variation in $u_{z}$ is small, due to the fact that close to the surface the vertical component of velocity must be small. As a consequence, the interaction term $\omega_{y} \partial_{y} u_{z}$ in equation (15), which acts to tilt lateral vorticity in the vertical direction, will be large for the leeward fire case and negligibly small in the windward fire case.

\section{RESULTS}

\subsection{Fire on a leeward slope}

Focusing on the right flank of the fire, the tilting term $\omega_{y} \partial_{y} u_{z}$ is positive and so, according to (15), acts to convert positive lateral vorticity $\omega_{y}$ into positive vertical vorticity $\omega_{z}$. Once positive $\omega_{z}$ is generated, equation (15) implies that the buoyant plume will further enhance $\omega_{z}$ through the stretching term $\omega_{z} \partial_{z} u_{z}$. Conversely, on the left flank the terms act in the same way to produce negative $\omega_{z}$.

Considering equation (13), once vertical vorticity $\omega_{z}$ is generated through tilting of lateral vorticity by the flow of the buoyant plume, and subsequent enhancement by vertical stretching, a component will be tilted into longitudinal vorticity $\omega_{x}$ by the (separated) ambient flow. This will be in addition to the longitudinal vorticity that may be produced through tilting of $\omega_{y}$ if $\partial_{y} u_{x}$ is non-zero. Additional $\omega_{x}$ will be generated by baroclinic torque through the $\partial_{y} \rho \partial_{z} p$ term. Generation of positive $\omega_{x}$ on the right flank and negative $\omega_{x}$ on the left flank will be compensated to some extent by negative stretching on both flanks by the $\omega_{x} \partial_{x} u_{x}$ term in (13).

The net effect implied by equations (13) and (15) is that initial tilting of lateral vorticity by the pyrogenic flow will result in sustained generation of vertical and longitudinal vorticity as a fire burns on the lee slope. We will refer to the combination of $\omega_{x}$ and $\omega_{z}$ as the pyrogenic vorticity $\omega_{p}=\left(\omega_{x}, 0, \omega_{z}\right)$. This pyrogenic vorticity will manifest as a counter-rotating vortex pair on the two flanks of the fire with positive $\omega_{x}$ and $\omega_{z}$ on the right flank and negative $\omega_{x}$ and $\omega_{z}$ on the left flank. In each case this means that the vortex will be titled in the downwind direction. This is consistent with observations of experimental fires such as that shown in Figure 1. We note that more intense fires will produce stronger pyrogenic vorticity $\boldsymbol{\omega}_{p}$ due to the effects of the pyrogenic gradients $\partial_{y} u_{z}, \partial_{y} \rho$ and possibly $\partial_{y} u_{x}$.

On each flank the vortex would act to advect heat laterally across the top of the lee slope, thereby promoting the propagation of fire across the top of the leeward slope.

\subsection{Fire on a windward slope}

The lack of elevated positive lateral vorticity on the windward slope means that there is less propensity for $\omega_{y}$ to be tilted into $\omega_{z}$ in equation (15). As such, significant vertical vorticity is less likely on the windward side compared to the leeward side, in accordance with the observations of Countryman (1971). Equation (13) suggests that pyrogenic vorticity in the windward environment is more likely to be dominated by longitudinal vorticity $\omega_{x}$ driven by baroclinic torque. Significant vertical vorticity would not be expected until the fire crossed the ridge onto the leeward slope. The same conclusions would apply to fires burning on flat ground.

\section{DISCUSSION AND CONCLUSIONS}

This paper has presented simplified qualitative analyses of the vorticity equation, which examine the generation of pyrogenic vorticity in the immediate lee of a ridge. The cases of a fire originating from the leeward and windward slopes have been considered. The analyses indicated that vertical vorticity is generated on the flanks of the fire due to the tilting of ambient lateral vorticity by the updraft produced by the fire, and that this vertical vorticity can then be enhanced through vortex stretching. Vertical vorticity arising in this way manifests as a counter-rotating pair of vortices oriented in such a way as to advect hot air laterally across the top of the lee side of the ridge. This is consistent with patterns of fire spread showing rapid lateral spread across the top of steep leeward slopes (Sharples et al., 2012; Raposo et al., 2015) and with the results of numerical simulations that show an association between the atypical spread and the presence of vertical vorticity (Simpson et al., 2013). We note however, that in practice the vortex pairs are not always present (e.g. Figure 1): this suggests that there are other factors affecting vorticity tendency that are not represented in the present analysis. It is also worth noting that in many wildfire observations and simulations of atypical lateral spread, the fire propagates across the leeward slope as a very narrow finger constrained to the very top part of the leeward slope. This part of the slope appears to coincide with the region dominated by positive lateral vorticity.

The analyses also suggested the generation of horizontal (longitudinal) vorticity on the flanks of the fire, with 
contributions from tilting of ambient lateral vorticity and induced vertical vorticity, in addition to contributions from baroclinic torque and compensations due to negative stretching.

The analyses presented above, while rather crude, serve to extend the two-dimensional notions considered by Byron-Scott (1990), and provide some initial theoretical insights into the vorticity dynamics that appear to drive atypical lateral fire propagation. It is hoped that further work involving more sophisticated analyses of the vorticity equations will provide more detailed insights, for example into how how the generation of strong vertical vorticity at altitude produces strong along ridge surface winds.

\section{ACKNOWLEDGEMENT}

We acknowledge the anonymous reviewers whose comments significantly improved the paper. J.J. Sharples and C.C. Simpson are supported by the Australian Research Council under the Discovery Indigenous Project IN130100038. The experimental fire in Figure 1 was conducted by Jason Forthofer, U.S. Fire Lab.

\section{REFERENCES}

Byron-Scott, R. (1990). The effects of ridge-top and lee-slope fires upon rotor motions in the lee of a steep ridge. Mathematical and Computer Modelling 13, 103-112.

Countryman, C. M. (1971). Fire whirls... why, when and where? Technical report, USDA Forest Service, Pacific Southwest Forest and Range Experiment Station, Berkeley, CA.

Cruz, M., A. Sullivan, J. Gould, N. Sims, A. Bannister, J. Hollis, and R. Hurley (2012). Anatomy of a catastrophic wildfire: the Black Saturday Kilmore East fire in Victoria, Australia. Forest Ecology and Management 284, 269-285.

Holton, J. R. and G. J. Hakim (2012). An introduction to dynamic meteorology, Volume 88. Academic press.

Jenkins, M. A., T. Clark, and J. Coen (2001). Coupling atmospheric and fire models. In E. A. Johnson and K. Miyanishi (Eds.), Forest Fires, Behavioural and Ecological Effects, pp. 257-302. Academic Press: San Diego, CA, USA.

Klemp, J. B. (1987). Dynamics of tornadic thunderstorms. Annual Review of Fluid Mechanics 19, 369-402.

Peterson, D. A., E. J. Hyer, J. R. Campbell, M. D. Fromm, J. W. Hair, C. F. Butler, and M. A. Fenn (2015). The 2013 Rim Fire: Implications for predicting extreme fire spread, pyroconvection, and smoke emissions. Bulletin of the American Meteorological Society 96, 229-247.

Raposo, J. R., S. Cabiddu, D. X. Viegas, M. Salis, and J. Sharples (2015). Experimental analysis of fire spread across a two-dimensional ridge under wind conditions. International Journal of Wildland Fire In press.

Sharples, J. J., R. H. D. McRae, and R. O. Weber (2010). Wind characteristics over complex terrain with implications for bushfire risk management. Environmental Modelling \& Software 25, 1099-1120.

Sharples, J. J., R. H. D. McRae, and S. R. Wilkes (2012). Wind-terrain effects on the propagation of large wildfires in rugged terrain: fire channelling. International Journal of Wildland Fire 21, 599-614.

Simpson, C. C., J. J. Sharples, and J. P. Evans (2015). Numerical modelling of wildland fire spread on the windward and leeward sides of a ridge. In Proceedings of MODSIM 2015. Modelling and Simulation Society of Australia and New Zealand.

Simpson, C. C., J. J. Sharples, J. P. Evans, and M. F. McCabe (2013). Large eddy simulation of atypical wildland fire spread on leeward slopes. International Journal of Wildland Fire 22, 282-296.

Vallis, G. K. (2006). Atmospheric and oceanic fluid dynamics: fundamentals and large-scale circulation. Cambridge University Press.

Wood, N. (1995). The onset of separation in neutral, turbulent flow over hills. Boundary-Layer Meteorology $76,137-164$. 